\title{
Subdiffusive dynamics and critical quantum correlations in a disorder-free localized Kitaev honeycomb model out of equilibrium
}

\author{
Guo-Yi Zhu $\circledast^{*}$ and Markus Heyl 10 \\ Max Planck Institute for the Physics of Complex Systems, Nöthnitzer Straße 38, Dresden 01187, Germany
}

(Received 21 December 2020; accepted 20 August 2021; published 17 September 2021)

\begin{abstract}
Disorder-free localization has recently emerged as a mechanism for ergodicity breaking in homogeneous lattice gauge theories. In this work we show that this mechanism can lead to unconventional states of quantum matter as the absence of thermalization lifts constraints imposed by equilibrium statistical physics. We study a Kitaev honeycomb model in a skew magnetic field subject to a quantum quench from a fully polarized initial product state and observe nonergodic dynamics as a consequence of disorder-free localization. We find that the system exhibits a subballistic power-law entanglement growth and quantum correlation spreading, which is otherwise typically associated with thermalizing systems. In the asymptotic steady state the Kitaev model develops volume-law entanglement and power-law decaying dimer quantum correlations even at a finite energy density. Our work sheds light on the potential for disorder-free localized lattice gauge theories to realize quantum states in two dimensions with properties beyond what is possible in an equilibrium context.
\end{abstract}

DOI: 10.1103/PhysRevResearch.3.L032069

\section{INTRODUCTION}

It is the general expectation that realistic isolated quantum many-body systems driven out of equilibrium eventually thermalize such that the relaxed long-time steady states become locally indistinguishable from thermal ensembles [1-6]. Two types of celebrated exceptions beyond this paradigm are quantum integrable models [7-11] and the Anderson or many-body localization (MBL) mechanism imposed by strong disorder $[12,13]$. In two dimensions, the exploration of ergodicity breaking dynamics in interacting systems remains a challenge especially in view of the argued instability of MBL in two dimensions [14]. Recent years have witnessed a new type of mechanism for nonergodic dynamics unique to lattice gauge theories where static local gauge charge or flux serves as a source for an effective internal disorder [15-17]. Importantly, this so-called disorder-free localization scenario does not rely on breaking translational invariance and can even occur in interacting two-dimensional (2D) models [18,19], opening up a promising route targeting the challenge of realizing quantum states in 2D nonergodic systems with properties beyond any equilibrium counterpart.

In this work we show that the Kitaev honeycomb model driven to highly excited states by a nonequilibrium quantum quench enters a peculiar disorder-free localized phase exhibiting subdiffusive dynamics towards a critical state exhibiting an algebraically decaying dimer quantum correlation func-

\footnotetext{
*timeexplorer1991@gmail.com
}

tion. Specifically, we investigate the nonequilibrium dynamics in the Kitaev honeycomb model in a weak skew magnetic field starting from a spin-polarized initial state. The problem can be mapped to a weakly interacting Majorana fermion model coupled to a static $\mathbb{Z}_{2}$ gauge field [20], which for the considered dynamics becomes effectively disordered. Although a number of previous works have considered the intertwined physics between fermion and flux in the Kitaev model [21-29], the central open question has remained as to whether this model can break ergodicity and can potentially host nonthermal quantum order. In the noninteracting limit, we find that the gauge flux disorder localizes most of the Majorana fermions but fails to freeze the metallic and critical modes, leading to the observed subdiffusive dynamics although the system is overall nonergodic [30-34]. We identify the subdiffusive dynamics in both an algebraic spread of quantum correlations and the power-law growth of entanglement. At late times, the system relaxes to a steady state with dimer quantum correlation functions decaying algebraically in space, which is characteristic of quasi-long-range order not accessible in thermal equilibrium. We argue that this quasi-long-range order implies a divergent multipartite entanglement as quantified by the quantum Fisher information. We find evidence that our main findings are robust against the leading-order perturbative Majorana fermion interactions induced by the skew magnetic field according to our numerical calculations for up to 128 spins on long timescales. Our results can be extended to any $\mathbb{Z}_{2}$ lattice gauge theory coupled to chiral Majorana fermions as long as the gauge flux can be considered static and disordered on the considered timescales.

\section{MODEL}

The Kitaev model consists of spin- $\frac{1}{2}$ degrees of freedom on the honeycomb lattice with spin-orbital locking Ising interactions $D_{j}^{\mu}=-\sigma_{j}^{\mu} \sigma_{j+e_{\mu}}^{\mu}$, where $j$ labels the spin site and $e_{\mu}$ 

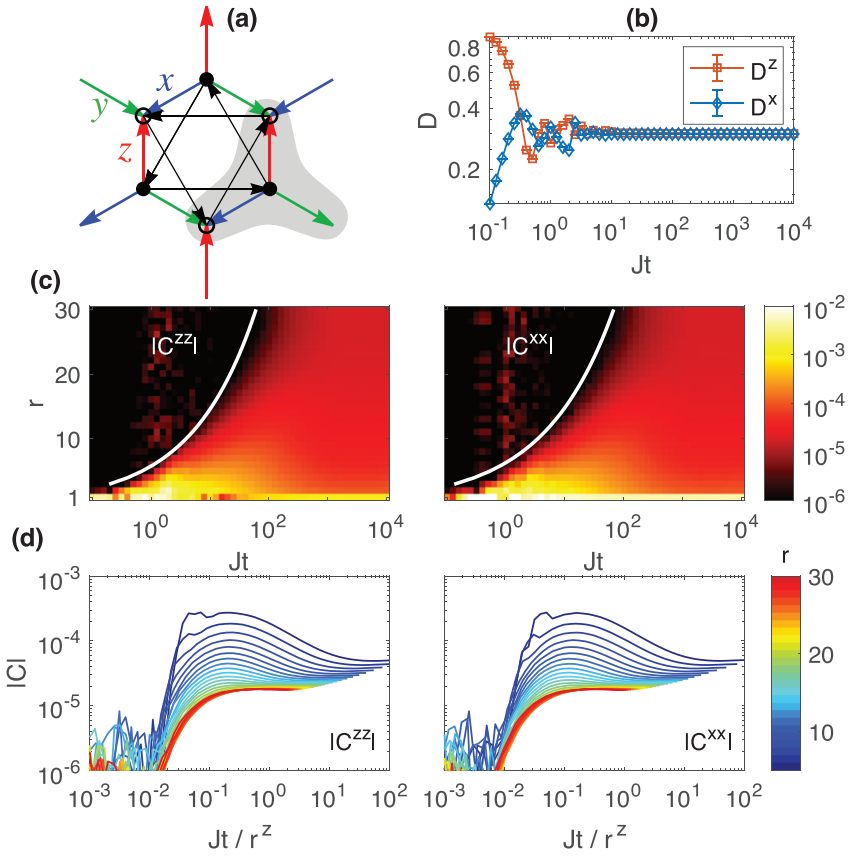

FIG. 1. (a) Model interactions. Arrows indicate Majorana fermion hopping. Four Majorana fermions interact within every $\mathrm{Y}$ junction, shaded in gray. (b) $\left\langle D^{z(x)}(t)\right\rangle$. (c) $C^{\mu \mu}=\left\langle D_{j}^{\mu} D_{j+r}^{\mu}\right\rangle_{c}$. The propagating wave front is determined by the threshold $|C| \geqslant 10^{-6}$, subject to a power-law fitting $r \propto(J t)^{1 / z}$ indicated by white lines. Here $z=2.5(2)$ for $\left|C^{z z}\right|$ and $z=2.7(3)$ for $\left|C^{x x}\right|$. (d) Collapse of correlation growth at fixed distance with rescaled time. The parameters are $J_{z}=J_{x}=J_{y}=J, \tilde{h}=0.25 \mathrm{~J}$, and 10000 disorder samples of system size with $60 \times 60$ unit cells (7200 spins) have been used.

denotes the nearest-neighbor vector of different orientations $\mu=x, y, z$ [Fig. 1(a)]. In the presence of a weak [111] skew magnetic field the Hamiltonian is

$$
\hat{H}_{K}=\sum_{j} \sum_{\mu=x, y, z}\left(J_{\mu} \sigma_{j}^{\mu} \sigma_{j+e_{\mu}}^{\mu}+h \sigma_{j}^{\mu}\right), \quad h \ll J_{\mu} .
$$

For $h=0$ the product $\hat{W} \equiv D^{x} D^{y} D^{z} D^{x} D^{y} D^{z}$ surrounding a hexagon plaquette commutes with $\hat{H}_{K}$, implying an extensive number of local integrals of motion. In the targeted limit $h \ll J_{\mu}$ we take into account the magnetic field perturbatively to the leading order that preserves these local symmetries [20],

$$
\hat{H}=\sum_{\mu} \sum_{j} J_{\mu} \sigma_{j}^{\mu} \sigma_{j+e_{\mu}}^{\mu}+\tilde{h} \sum_{(i j k) \in \wedge, Y} \sigma_{i}^{x} \sigma_{j}^{y} \sigma_{k}^{z},
$$

where $\tilde{h} \propto h^{3} / J^{2}$. The perturbative interaction acts on three spins that exist on any wedges $\wedge$ or the end of any $Y$ junction. Either by introducing the gauge redundancy [20] and fixing the gauge or by a Jordan-Wigner transformation [35,36], one can map $\hat{H}$ onto an interacting Majorana fermion minimally coupled with $\mathbb{Z}_{2}$ gauge field on the links,

$$
\begin{aligned}
\hat{H}= & \sum_{\langle j \rightarrow l\rangle} J_{\mu} i u_{j, l} \beta_{j} \alpha_{l}+\tilde{h} \sum_{\langle j \rightarrow l\rangle} i u_{j, k} u_{k, l}\left(\alpha_{j} \alpha_{l}+\beta_{j} \beta_{l}\right) \\
& +\tilde{h} \sum_{\mathrm{Y}} u_{i, j} u_{i, k} u_{i, l}\left(\beta_{i} \alpha_{j} \alpha_{k} \alpha_{l}-\alpha_{i} \beta_{j} \beta_{k} \beta_{l}\right),
\end{aligned}
$$

where $\alpha(\beta)$ denotes Majorana fermion on the $A(B)$ sublattice marked with open (closed) circles in Fig. 1(a). The static gauge field on the link is pinned to $u_{j, j-e_{x(y)}}=1$ and $u_{j, j-e_{z}}= \pm 1$. The $\hat{W}$ on plaquettes are transformed to be locally conserved $\mathbb{Z}_{2}$ gauge fluxes. The last four-fermion term is a chiral and gauged Majorana Hubbard interaction [37], where $j, k$, and $l$ are arranged in counterclockwise order around $i$.

\section{QUANTUM QUENCH PROTOCOL}

We prepare a simple initial state as a Néel state such that $\sigma^{z}\left|\Psi_{0}\right\rangle= \pm\left|\Psi_{0}\right\rangle$ on the $A$ or $B$ sublattice, respectively, which is to be evolved by $\hat{H}$ later on. In the fermion representation the initial state becomes a gauged fermion vacuum coupled to a disordered gauge-field background

$$
|\Psi(t)\rangle=\frac{1}{2^{N / 2}} \sum_{\{u\}} e^{-i t \hat{H}_{[u]}}|\{u\}\rangle \otimes\left|\psi_{\{u\}}\right\rangle,
$$

where $N$ is the number of unit cells ( $z$ links) and the Fock state satisfies $i u_{j, j-e_{z}} \alpha_{j} \beta_{j-e_{z}}\left|\psi_{\{u\}}\right\rangle=\left|\psi_{\{u\}}\right\rangle$. In the sector $\prod_{j} \sigma_{j}^{z}=$ 1 the antiperiodic boundary condition in the spin Hamiltonian is mapped to the periodic boundary in the Majorana Hamiltonian. Here we will mainly focus on the isotropic coupling $J_{x}=J_{y}=J_{z} \equiv J$ and $\tilde{h}=0.25 J$.

For observables that preserve the gauge field $\hat{O}=$ $\sum_{\{u\}} O_{\{u\}}[15,16]$,

$$
\left\langle\Psi_{0}|\hat{O}(t)| \Psi_{0}\right\rangle=\frac{1}{2^{N}} \sum_{\{u\}}\left\langle\psi_{\{u\}}\left|e^{i t \hat{H}_{[u\}}} \hat{O}_{\{u\}} e^{-i t \hat{H}_{[u u} \mid}\right| \psi_{\{u\}}\right\rangle,
$$

where the average over gauge-field configurations can be performed via Monte Carlo sampling. The typical $\{u\}$ configuration is random, making the dynamical problem equivalent to Majorana fermions subject to $\mathbb{Z}_{2}$ gauge $(\pi)$ flux disorder, although our model is overall translationally invariant $[15,16]$.

Overall we target the description of the nonequilibrium dynamics through a sequence of two steps. First, we will study in detail the exact solvable point, i.e., the noninteracting limit of Eq. (3), where we find that the system becomes nonergodic due to disorder-free localization, and afterward explore the influence of interactions.

\section{EXACTLY SOLVABLE POINT}

When the Majorana interactions are neglected, the model becomes exactly solvable. For each gauge configuration the dynamics is governed by a free Majorana fermion Gaussian Hamiltonian that can be computed efficiently. By randomly sampling gauge fields $u= \pm 1$ on the $z$ links, we compute the real-time evolution of various physical observables that are natural in both the spin and the fermion language.

First, we consider the spin dimer expectation values $D_{j}^{\mu}=$ $i u_{j, j-e_{\mu}} \alpha_{j} \beta_{j-e_{\mu}}(\mu=z, x)$, which relax exponentially fast to the same constant losing the memory of initial anisotropy [see Fig. 1(b)]. The observables along the $x$ and $y$ directions can be related by mirror symmetry. As we will show, the dimer quantum correlation functions $C^{z z(x x)}(r, t)=$ $\frac{1}{N} \sum_{j}\left\langle\Psi(t)\left|D_{j}^{z(x)} D_{j+r}^{z(x)}\right| \Psi(t)\right\rangle_{c}$ exhibit a much slower and intricate dynamics, which quantifies the correlation of gauged local fermion parity. Remarkably, we find that $C^{z z(x x)}(r, t)$ 


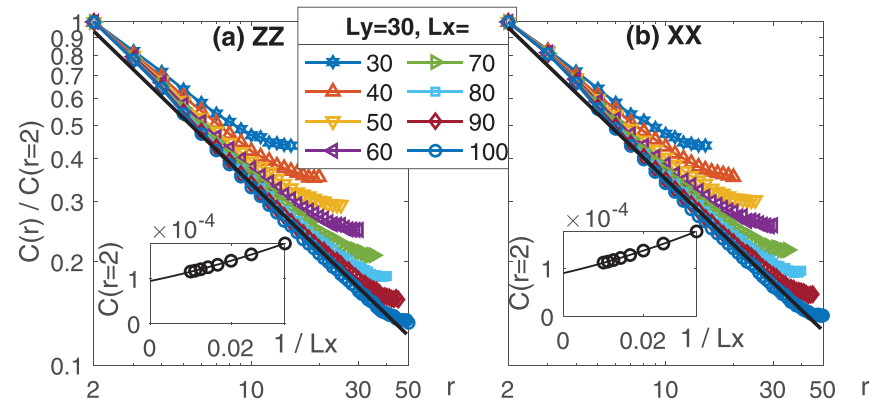

FIG. 2. Dimer correlations in the steady state, averaged over $10^{3.6} \lesssim J t \lesssim 10^{4}$. Black lines indicate power-law fitting $r^{-\Delta}$ with $\Delta=0.63(2)$ for both $C^{z z}$ and $C^{x x}$. Insets show correlations at $r=2$ extrapolated to finite values in the thermodynamic limit.

exhibits an algebraic light cone in space-time [see Fig. 1(c)], with the wave front following a power-law $J t \propto r^{z}$ behavior. The dynamical exponent is obtained as $z=2.5(2)$ for $\left|C^{z z}\right|$ and $z=2.7(3)$ for $\left|C^{x x}\right|$. Notice that the dynamical exponent $z$ here is associated with information transport instead of particle or energy transport, and $z>1$ signals subdiffusion [38]. In Fig. 1(d) we further corroborate this by achieving a data collapse upon rescaling the time axis $J t / r^{z}$. While such subballistic behavior in systems with conventional disorder is typically observed on the ergodic side close to the MBL transition lying between diffusive and glassy limits [30-34], here we observe such dynamics for a disorder-free localized model, as we will argue in more detail below.

At long times the system settles to a steady state, which, as we find, is of nonergodic critical nature with correlations decaying algebraically in space, as seen in Fig. 2. We observe that the decay of $C^{z z(x x)}(r, t)$ is consistent with a power law in space, whose exponent increases for larger system sizes and appears to converge near $0.63(2)$. The power-law decaying correlation function in all directions $x, y$, and $z$ is reminiscent of the Kosterlitz-Thouless phase with quasi-long-range order [39], without spontaneously breaking the spin-orbital threefold rotation symmetry. However, even when this symmetry is explicitly broken in the anisotropic regime, we still observe a critical correlation [40]. It is the effective disorder that partially inhibits the finite-energy density fluctuations and stabilizes the quasi-long-range spin dimer order [41].

These critical quantum correlations further have an immediate impact on the entanglement content of the reached steady state, as the dimer quantum correlation function can be directly linked to a quantum Fisher information density via $f_{Q}^{z z(x x)}(t)=\sum_{r} C^{z z(x x)}(r, t)[42-44]$. Since $C^{z z(x x)}(r, t) \sim r^{-\Delta}$ with $0<\Delta<1$ for $t \rightarrow \infty$, we find that $f_{Q}^{z z(x x)} \sim N^{1-\Delta}$ diverges in the thermodynamic limit. As a consequence, the steady state exhibits strong multipartite entanglement.

For a more detailed quantification of the entanglement properties we further consider the projective bipartite entanglement entropy that serves as an entanglement diagnostic for quantum disentangled liquids [45-47]. Namely, we measure the von Neumann entanglement entropy for half of the Majorana fermions, when the gauge field is projected onto the

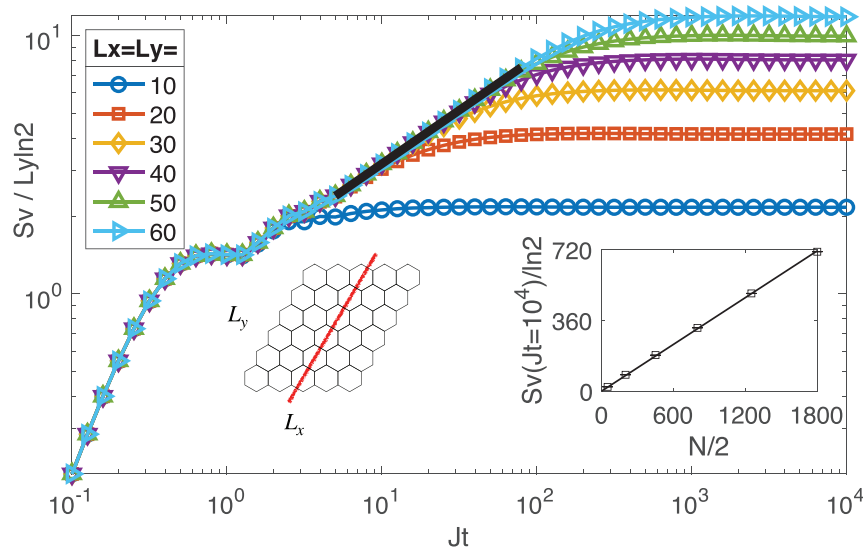

FIG. 3. Projective bipartite entanglement entropy, averaged over 1000 disorder samples. The inset shows the entanglement cut. The black line indicates the power-law fitting proportional to $t^{1 / z}$ with $z=2.4(1)$. At late time, entropy saturates to a volume law $S_{v}(\mathrm{~J} t=$ $\left.10^{4}\right)=0.40(1) N \ln 2 / 2$, as shown in the inset.

diagonal ensemble

$$
S_{v}=\frac{1}{2^{N}} \sum_{\{u\}} S_{\{u\}}, \quad S_{\{u\}}=-\hat{\rho}_{\{u\}} \ln \hat{\rho}_{\{u\}},
$$

with the reduced density matrix

$$
\hat{\rho}_{\{u\}}=\operatorname{Tr}_{\alpha, \beta \in L} e^{-i t \hat{H}_{\{u\}}}\left|\psi_{\{u\}}\right\rangle\left\langle\psi_{\{u\}}\right| e^{i t \hat{H}_{\{u\}}}
$$

obtained from tracing out Majorana fermions on the left half of the lattice (see the inset in Fig. 3). Here $\hat{\rho}_{\{u\}}$ is a Gaussian operator which can be computed exactly $[48,49]$. Note that while we can compute local observables and correlation functions exactly, the nonprojective von Neumann entropy of the Kitaev model is not diagonal with respect to the gauge configurations and therefore cannot be reduced to a free fermion problem, unlike the low-order Rényi entropy [50]. Diagonal entanglement entropies such as the one we consider have been used already for localized systems in other contexts [51] and give an upper bound on the actual entanglement entropy [52]. As shown in Fig. 3, at early times the entanglement grows with an area law. At a second stage, the entanglement entropy exhibits a further growth according to a subballistic power law $S \propto t^{z}$. From a fit to the data we obtain the entanglement dynamical exponent $z=2.4(1)$, which within the accuracy of our simulations aligns with the exponent appearing for the subballistic spreading in $C^{z z(x x)}(r, t)$. In a system of finite size, we find that the entanglement entropy saturates to a volumelaw state $S_{v} \propto L_{x} L_{y}$ typical for the highly excited free fermion states $[53,54]$, as shown in the inset of Fig. 3. These numerical findings again highlight the unconventional nonequilibrium dynamics that we observe in the disorder-free localized Kitaev model.

One may ask what if we deform the initial state. By tuning the gauge flux density in the initial state by applying an operator $\prod_{q}\left[\frac{1}{2}+\left(\frac{1}{2}-p\right) \hat{W}_{q}\right]$, the exponent $\Delta$ as well as $z$ changes continuously, as visible in Fig. 4, which corroborates the robustness of the critical dynamical phase reminiscent of Kosterlitz-Thouless phase. However, notice that our critical dynamical phase at late-time steady state should be contrasted 

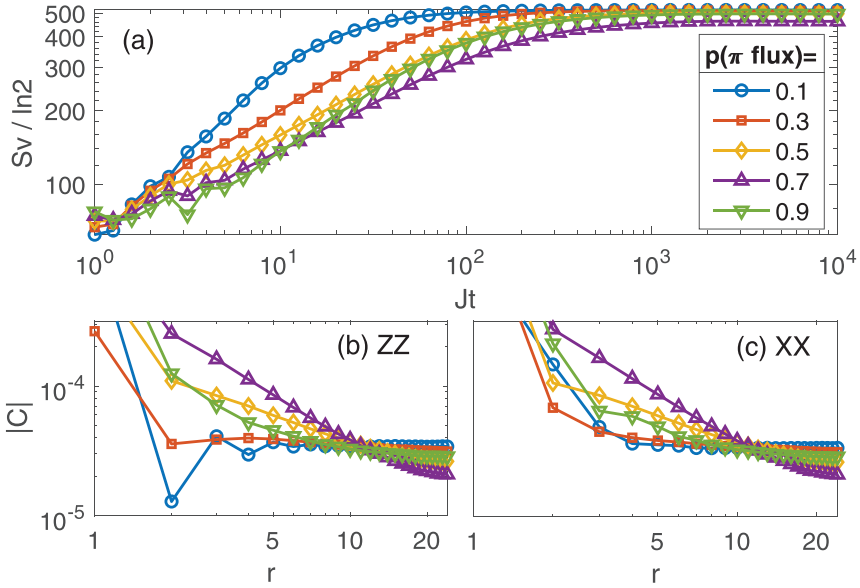

FIG. 4. Tuning the density of $\pi$ fluxes. (a) Projective bipartite entanglement entropy of Majorana fermions. (b) and (c) Steady-state spatial correlation function $\left|C^{z z(x x)}\right|$, averaged in the time window $10^{3} \lesssim J t \lesssim 10^{4}$. The parameters are $J_{z}=J_{x}=J_{y} \equiv J, \tilde{h}=0.25 J$, $L_{x}=L_{y}=50$, and 1000 disorder samples have been used.

with the one exhibiting critical initial slip in short-time relaxation [55].

\section{LOCALIZATION ANALYSIS}

The peculiar coexistence of subdiffusive dynamics at transient time and the quasi-long-range order at late time implies a subtle localization scenario in behind. Indeed, we find a mixture of localized and critical modes from the standard numerical diagnostics [40] including level spacing statistics [56,57], 2D localization length [58-60], and Chern number [20,61-63].

The localization length is calculated by the retarded Green's function using the iterative Dyson equation for a semi-infinite quasi-one-dimensional geometry, followed by a one-parameter scaling collapse for varying narrow width. As shown in Fig. 5(a), the localization length and the level
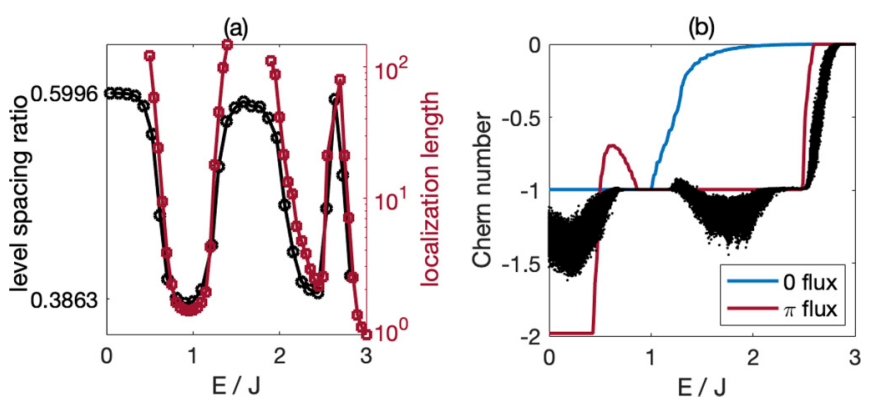

FIG. 5. (a) The left axis shows the level spacing ratio, for system size $L_{x}=L_{y}=60$ with 10000 disorder samples. The characteristic value for the Poisson ensemble, $2 \ln 2-1 \simeq 0.3863$, and the one for the Gaussian unitary ensemble, approximately equal to 0.5996 , are indicated. The right axis shows the localization length obtained by one-parameter scaling for a sequence of quasi-1D long stripes for $L_{y}=8,16,32,64,128$ and $L_{x} \leqslant 10^{6}$. (b) Chern number for system size $L_{x}=L_{y}=40$. Black dots are for 500 disorder samples, while the blue (red) line is for the zero $(\pi)$ flux clean system. spacing ratio for a finite-size system are consistent in showing three energy windows with delocalization tendency. The delocalization at zero energy was known to be responsible for a low-energy Majorana thermal metal state in the class D dirty superconductors [64-66] or Majorana lattice model [67-69], which entails a logarithmically divergent density of states and weak multifractal nature as we numerically verify $[40,70]$. Intuitively, the low-energy delocalized Majorana mode arises from percolating through an extensive number of resonating Majorana zero modes trapped in $\mathbb{Z}_{2}$ gauge fluxes in a weakpairing topological superconductor [68,71].

To gain more insight into the topology of the fermion, we calculate the Chern number of the fermion eigenstates [72-74] of varying energy by using the real-space formula based on the concept of a noncommutative Brillouin zone:

$$
C=\frac{2 \pi i}{N} \operatorname{Tr}([P x P, P y P]), \quad P(E)=\sum_{\epsilon<-E}|\epsilon\rangle\langle\epsilon| .
$$

Here $x$ and $y$ are the real-space coordinate operators which label the first quantized orbitals and generate the translation of crystal momenta, $|\epsilon\rangle$ is the single-particle eigenstate of the first quantized Hamiltonian matrix with energy $\epsilon$, and $P(E)$ is the spectral projector where the single-particle mode with energy smaller than $-E$ is occupied, mimicking the Fermi level in the complex fermion system with number conservation. In a fermionic system with only fermion parity conservation, half of the single-particle eigenstates are redundant, so we consider only $E \geqslant 0$. The change of $C(E)$ reveals the Berry flux carried by the fermion mode at the corresponding energy. From Fig. 5(b), the delocalized mode near $E \simeq 2.5(1) \mathrm{J}$ is clearly associated with a topological quantum critical point separating two distinct Chern plateaus that is robust against perturbation and weak disorder [75,76]. As for the energy window $1.5 \mathrm{~J} \lesssim E \lesssim 2.0 \mathrm{~J}$, it is unclear whether it would maintain a finite mobility edge or shrink to a singular point or become fully localized in the thermodynamic limit $[77,78]$.

A final comment is that our result is consistent with the argument that non-Abelian topological phases cannot be fully localized [79]. While disorder tends towards localization, i.e., a divergent dynamical exponent $z \rightarrow \infty$, this tendency competes with the metallic and topology induced critical modes favoring ballistic propagation with $z=1$, leading to the observed subdiffusive dynamics with $z>1$.

\section{BEYOND EXACT SOLVABLE LIMIT}

Now we aim to address the robustness of our observations upon the influence of interactions present in Eq. (3). Here we will focus on the leading-order resonant contributions responsible for an eventual destabilization, by utilizing the approach introduced in Ref. [80], where it has been shown that these resonant contributions can capture the essential nonperturbative effects of interactions such as the logarithmic entanglement growth in MBL phases not only on a qualitative but also on a quantitative level. The Hamiltonian is then expressed in the canonical fermion basis

$$
\hat{H}_{\gamma}=-\sum_{n=1}^{N} \epsilon_{n} i \gamma_{n}^{\prime} \gamma_{n}^{\prime \prime}-\frac{1}{4} \sum_{m, n=1}^{N} V_{m, n} \gamma_{m}^{\prime} \gamma_{m}^{\prime \prime} \gamma_{n}^{\prime} \gamma_{n}^{\prime \prime}+\cdots,
$$


where the canonical Majorana fermions $\gamma^{\prime}$ and $\gamma^{\prime \prime}$ are related to the original local Majorana fermions by an orthogonal transformation obtained from diagonalizing the noninteracting fermion part. The leading-order resonant interaction preserves the parity of the canonical fermion mode $\left\langle i \gamma_{n}^{\prime} \gamma_{n}^{\prime \prime}\right\rangle$ but induces a dephasing effect, analogous to the $l$-bit theory in MBL systems $[12,13]$ in which it leads to dramatic nonperturbative effect [80]. In our case of the Kitaev model with weak magnetic field, we also find a special structure for this interaction strength $V_{m, n}$, which endows a hierarchy of dephasing timescales [see Fig. 6(a)]. It is the key observation in Ref. [80] that the dynamics of any fermion correlation function can be effectively written as a sum over a number $O\left(N^{4}\right)$ of Gaussian evolution trajectories, schematically abbreviated as

$$
\begin{aligned}
& \left\langle\psi(t)\left|\gamma_{m} \gamma_{n} \gamma_{p} \gamma_{q}\right| \psi(t)\right\rangle \\
& \quad=\sum_{m n p q} C\left\langle\psi\left|e^{-i t(1 / 4) \gamma A_{m n p q} \gamma} \gamma_{m} \gamma_{n} \gamma_{p} \gamma_{q}\right| \psi\right\rangle,
\end{aligned}
$$

which can be further factorized into the product of a Loschmidt amplitude quantity and an effective correlation function [40,81-85]. As shown in Fig. 6(d), we calculate the system with $16 \times 4$ unit cells (128 spins) up to the timescale $J t \leqslant 10^{4}$, where the interacting scenario turns out to collapse with the noninteracting case within numerical accuracy. The critical quantum correlations are therefore stable up to this timescale. We estimate the validity of the perturbative approach by the statistics of resonances in first-order perturbation theory of the omitted terms. We find that they are off-resonant with probability $\gtrsim 99.5 \%$ for the considered parameter regime [40], above typical thresholds [86], so they can become relevant only via higher-order processes manifesting on longer timescales.

\section{CONCLUSION}

While thermalization may occur eventually for the full Hamiltonian in Eq. (1) on long timescales, we emphasize that our findings imply a long intermediate-time window with nonergodic behavior leading to exotic quantum dynamics and correlations. On the other hand, it is tempting to ask whether the exotic dynamics and nonequilibrium quantum order are related to the low-energy non-Abelian Ising topological order $[20,87]$. However, in the strongly anisotropic coupling regime which in zero temperature exhibits a distinct Abelian $\mathbb{Z}_{2}$ topological order [88], we find a similar high-energy critical mode and subdiffusive dynamics as well as critical correlation, which goes beyond the low-energy universality class [40]. Our findings of subdiffusive dynamics and critical quantum correlations may emerge universally in general $\mathbb{Z}_{2}$ lattice gauge theories coupled to chiral Majorana matter fermions, provided two essential ingredients: (i) nontrivial fermion topology and
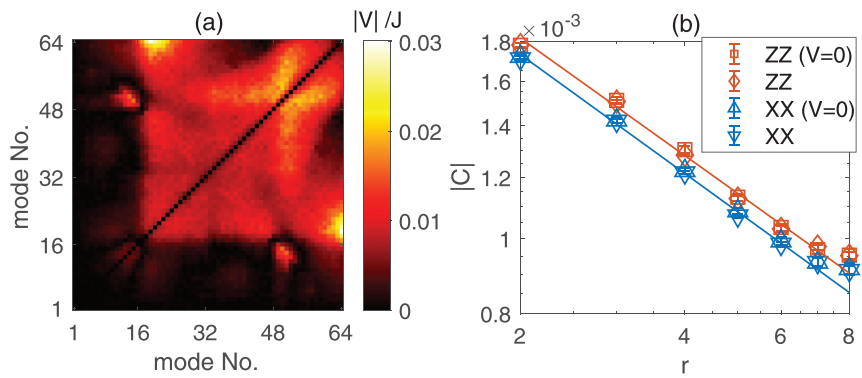

FIG. 6. (a) $V_{m n}$. The mode index corresponds to the energy in ascending order. (b) Correlation functions of steady state, averaged in the time window $10^{3} \lesssim J t \lesssim 10^{4}$. The power-law-fitting exponent is 0.50(6) for $C^{z z}$ and 0.51(4) for $C^{x x}$. The parameters are $\tilde{h}=0.25 \mathrm{~J}$, $L_{x}=16, L_{y}=4$, and 200 disorder samples. The $V=0$ case for comparison takes 10000 disorder samples.

(ii) static disordered gauge flux [89-92]. Above all, our observation of quasi-long-range order with associated divergent multipartite entanglement in a nonequilibrium high-energy steady state marks a concrete step towards yet unexplored unconventional phase structures in ergodicity breaking 2D quantum models. Subdiffusion might be present also in a more general context of Majorana spin liquids as long as the effective $\mathbb{Z}_{2}$ gauge flux (vison) dynamics is much slower than that of the fermions, which will be a challenging but valuable scope for future research. Furthermore, motivated by the recent developments showing that the gauge charge disorder in a 1D unconstrained gauge theory can stabilize a time crystal order [93], it would also be interesting to generalize this idea to two dimensions in search of nontrivial spatiotemporal order from a driven Kitaev model $[94,95]$. Finally, beyond the conceptual interest, the nonequilibrium quench dynamics can in principle be realized in various quantum architectures including ultracold atoms [96,97], superconducting qubits [98,99], or topological nanowires [100,101], as well as via ultrafast pump-probe techniques in the Kitaev candidate materials at sufficiently low temperatures with suppressed phonon influence $[29,102-106]$.

\section{ACKNOWLEDGMENTS}

The authors would like to thank G. D. Tomasi, C. Castelnovo, and O. Hart for helpful discussions. G.-Y.Z. also thanks S.-K. Jian and C. Chen for inspiring discussions and the IT teams in MPIPKS and MPCDF for technical support in computations. This project has received funding from the $\mathrm{Eu}-$ ropean Research Council (ERC) under the European Unions Horizon 2020 research and innovation programme (Grant Agreement No. 853443). M.H. further acknowledges support from the Deutsche Forschungsgemeinschaft (DFG) via the Gottfried Wilhelm Leibniz Prize program.
[1] J. M. Deutsch, Quantum statistical mechanics in a closed system, Phys. Rev. A 43, 2046 (1991).

[2] M. Srednicki, Chaos and quantum thermalization, Phys. Rev. E 50, 888 (1994).
[3] M. Rigol, V. Dunjko, and M. Olshanii, Thermalization and its mechanism for generic isolated quantum systems, Nature (London) 452, 854 (2008) 
[4] J. Eisert, M. Friesdorf, and C. Gogolin, Quantum manybody systems out of equilibrium, Nat. Phys. 11, 124 (2015).

[5] J. M. Deutsch, Eigenstate thermalization hypothesis, Rep. Prog. Phys. 81, 082001 (2018).

[6] A. Mitra, Quantum quench dynamics, Annu. Rev. Condens. Matter Phys. 9, 245 (2018).

[7] P. Calabrese and J. Cardy, Evolution of entanglement entropy in one-dimensional systems, J. Stat. Mech. (2005) P04010.

[8] P. Calabrese and J. Cardy, Time-Dependence of Correlation Functions Following a Quantum Quench, Phys. Rev. Lett. 96, 136801 (2006).

[9] V. Alba and P. Calabrese, Entanglement and thermodynamics after a quantum quench in integrable systems, Proc. Natl. Acad. Sci. U.S.A. 114, 7947 (2017)

[10] J. E. Moore, A perspective on quantum integrability in manybody-localized and Yang-Baxter systems, Philos. Trans. R. Soc. A 375, 20160429 (2017).

[11] P. Calabrese, Entanglement spreading in non-equilibrium integrable systems, SciPost Phys. Lect. Notes 20 (2020).

[12] R. Nandkishore and D. A. Huse, Many body localization and thermalization in quantum statistical mechanics, Annu. Rev. Condens. Matter Phys. 6, 15 (2015).

[13] D. A. Abanin, E. Altman, I. Bloch, and M. Serbyn, Many-body localization, thermalization, and entanglement, Rev. Mod. Phys. 91, 021001 (2019).

[14] I.-D. Potirniche, S. Banerjee, and E. Altman, Exploration of the stability of many-body localization in $d>1$, Phys. Rev. B 99, 205149 (2019).

[15] A. Smith, J. Knolle, D. L. Kovrizhin, and R. Moessner, Disorder-Free Localization, Phys. Rev. Lett. 118, 266601 (2017).

[16] M. Brenes, M. Dalmonte, M. Heyl, and A. Scardicchio, ManyBody Localization Dynamics from Gauge Invariance, Phys. Rev. Lett. 120, 030601 (2018).

[17] H. Yarloo, A. Langari, and A. Vaezi, Anyonic self-induced disorder in a stabilizer code: Quasi many body localization in a translational invariant model, Phys. Rev. B 97, 054304 (2018).

[18] A. Smith, J. Knolle, R. Moessner, and D. L. Kovrizhin, Dynamical localization in $\mathbb{Z}_{2}$ lattice gauge theories, Phys. Rev. B 97, 245137 (2018).

[19] P. Karpov, R. Verdel, Y. P. Huang, M. Schmitt, and M. Heyl, Disorder-Free Localization in an Interacting TwoDimensional Lattice Gauge Theory, Phys. Rev. Lett. 126, 130401 (2021).

[20] A. Kitaev, Anyons in an exactly solved model and beyond, Ann. Phys. (NY) 321, 2 (2006).

[21] J. Knolle, D. L. Kovrizhin, J. T. Chalker, and R. Moessner, Dynamics of a Two-Dimensional Quantum Spin Liquid: Signatures of Emergent Majorana Fermions and Fluxes, Phys. Rev. Lett. 112, 207203 (2014).

[22] X.-Y. Song, Y.-Z. You, and L. Balents, Low-Energy Spin Dynamics of the Honeycomb Spin Liquid Beyond the Kitaev Limit, Phys. Rev. Lett. 117, 037209 (2016).

[23] J. Nasu, J. Yoshitake, and Y. Motome, Thermal Transport in the Kitaev Model, Phys. Rev. Lett. 119, 127204 (2017).

[24] A. Metavitsiadis, A. Pidatella, and W. Brenig, Thermal transport in a two-dimensional $\mathbb{Z}_{2}$ spin liquid, Phys. Rev. B 96, 205121 (2017).
[25] M. Gohlke, R. Moessner, and F. Pollmann, Dynamical and topological properties of the Kitaev model in a [111] magnetic field, Phys. Rev. B 98, 014418 (2018).

[26] J. Knolle, S. Bhattacharjee, and R. Moessner, Dynamics of a quantum spin liquid beyond integrability: The KitaevHeisenberg- $\Gamma$ model in an augmented parton mean-field theory, Phys. Rev. B 97, 134432 (2018).

[27] M. Gohlke, R. Verresen, R. Moessner, and F. Pollmann, Dynamics of the Kitaev-Heisenberg Model, Phys. Rev. Lett. 119, 157203 (2017).

[28] L. Rademaker, Quenching the Kitaev honeycomb model, SciPost Phys. 7, 071 (2019).

[29] J. Nasu and Y. Motome, Nonequilibrium Majorana dynamics by quenching a magnetic field in Kitaev spin liquids, Phys. Rev. Research 1, 033007 (2019).

[30] E. Altman and R. Vosk, Universal dynamics and renormalization in many body localized systems, Annu. Rev. Condens. Matter Phys. 6, 383 (2015).

[31] R. Vosk, D. A. Huse, and E. Altman, Theory of the ManyBody Localization Transition in One-Dimensional Systems, Phys. Rev. X 5, 031032 (2015).

[32] D. J. Luitz, N. Laflorencie, and F. Alet, Extended slow dynamical regime close to the many-body localization transition, Phys. Rev. B 93, 060201(R) (2016).

[33] D. J. Luitz and Y. Bar Lev, Information propagation in isolated quantum systems, Phys. Rev. B 96, 020406(R) (2017).

[34] T. L. M. Lezama and D. J. Luitz, Power-law entanglement growth from typical product states, Phys. Rev. Research 1, 033067 (2019).

[35] X.-Y. Feng, G.-M. Zhang, and T. Xiang, Topological Characterization of Quantum Phase Transitions in a Spin-1/2 Model, Phys. Rev. Lett. 98, 087204 (2007).

[36] D.-H. Lee, G.-M. Zhang, and T. Xiang, Edge Solitons of Topological Insulators and Fractionalized Quasiparticles in Two Dimensions, Phys. Rev. Lett. 99, 196805 (2007).

[37] A. Rahmani and M. Franz, Interacting Majorana fermions, Rep. Prog. Phys. 82, 084501 (2019).

[38] D. J. Luitz and Y. Bar Lev, The ergodic side of the manybody localization transition, Ann. Phys. (Berlin) 529, 1600350 (2017).

[39] J. M. Kosterlitz, The critical properties of the two-dimensional xy model, J. Phys. C 7, 1046 (1974).

[40] See Supplemental Material at http://link.aps.org/ supplemental/10.1103/PhysRevResearch.3.L032069 for the numerical results and technical details for the dynamics of the tuning flux density, other Majorana fermion correlations, the dynamics of anisotropic coupling case, the time-reversal symmetric case, the fermionization, the derivation of the time-evolved physical observables with and without interaction, the calculation of the localization length and Chern number, and evidence of the zero-energy Majorana metallic state.

[41] D. A. Huse, R. Nandkishore, V. Oganesyan, A. Pal, and S. L. Sondhi, Localization protected quantum order, Phys. Rev. B 88, 014206 (2013).

[42] P. Hyllus, W. Laskowski, R. Krischek, C. Schwemmer, W. Wieczorek, H. Weinfurter, L. Pezzé, and A. Smerzi, Fisher information and multiparticle entanglement, Phys. Rev. A 85, 022321 (2012). 
[43] G. Tóth, Multipartite entanglement and high-precision metrology, Phys. Rev. A 85, 022322 (2012).

[44] P. Hauke, M. Heyl, L. Tagliacozzo, and P. Zoller, Measuring multipartite entanglement via dynamic susceptibilities, Nat. Phys. 12, 778 (2016).

[45] T. Grover and M. P. A. Fisher, Quantum disentangled liquids, J. Stat. Mech. (2014) P10010.

[46] D. Ben-Zion, J. McGreevy, and T. Grover, Disentangling quantum matter with measurements, Phys. Rev. B 101, 115131 (2020).

[47] A. Smith, J. Knolle, R. Moessner, and D. L. Kovrizhin, Absence of Ergodicity without Quenched Disorder: From Quantum Disentangled Liquids to Many-Body Localization, Phys. Rev. Lett. 119, 176601 (2017).

[48] G. Vidal, J. I. Latorre, E. Rico, and A. Kitaev, Entanglement in Quantum Critical Phenomena, Phys. Rev. Lett. 90, 227902 (2003).

[49] I. Peschel, Calculation of reduced density matrices from correlation functions, J. Phys. A: Math. Gen. 36, L205 (2003).

[50] O. Hart, S. Gopalakrishnan, and C. Castelnovo, Logarithmic Entanglement Growth from Disorder-Free Localization in the Two-Leg Compass Ladder, Phys. Rev. Lett. 126, 227202 (2021).

[51] M. Serbyn, Z. Papić, and D. A. Abanin, Universal Slow Growth of Entanglement in Interacting Strongly Disordered Systems, Phys. Rev. Lett. 110, 260601 (2013).

[52] A. Polkovnikov, Microscopic diagonal entropy and its connection to basic thermodynamic relations, Ann. Phys. (NY) 326, 486 (2011).

[53] H.-H. Lai and K. Yang, Entanglement entropy scaling laws and eigenstate typicality in free fermion systems, Phys. Rev. B 91, 081110(R) (2015).

[54] C. H. Lee, P. Ye, and X.-L. Qi, Position momentum duality in the entanglement spectrum of free fermions, J. Stat. Mech. (2014) P10023.

[55] H. K. Janssen, B. Schaub, and B. Schmittmann, New universal short-time scaling behaviour of critical relaxation processes, Z. Phys. B 73, 539 (1989).

[56] V. Oganesyan and D. A. Huse, Localization of interacting fermions at high temperature, Phys. Rev. B 75, 155111 (2007).

[57] T. Devakul and D. A. Huse, Anderson localization transitions with and without random potentials, Phys. Rev. B 96, 214201 (2017).

[58] A. MacKinnon and B. Kramer, One-Parameter Scaling of Localization Length and Conductance in Disordered Systems, Phys. Rev. Lett. 47, 1546 (1981).

[59] A. MacKinnon and B. Kramer, The scaling theory of electrons in disordered solids: Additional numerical results, Z. Phys. B 53, 1 (1983).

[60] P. Markos, Numerical analysis of the anderson localization, Acta Phys. Slovaca 56, 561 (2006).

[61] J. Bellissard, A. van Elst, and H. S. Baldes, The noncommutative geometry of the quantum Hall effect, J. Math. Phys. 35, 5373 (1994).

[62] E. Prodan, T. L. Hughes, and B. A. Bernevig, Entanglement Spectrum of a Disordered Topological Chern Insulator, Phys. Rev. Lett. 105, 115501 (2010).

[63] R. Bianco and R. Resta, Mapping topological order in coordinate space, Phys. Rev. B 84, 241106(R) (2011).
[64] N. Read and D. Green, Paired states of fermions in two dimensions with breaking of parity and time-reversal symmetries, and the fractional quantum Hall effect, Phys. Rev. B 61, 10267 (2000).

[65] T. Senthil and M. P. A. Fisher, Quasiparticle localization in superconductors with spin-orbit scattering, Phys. Rev. B 61, 9690 (2000)

[66] J. T. Chalker, N. Read, V. Kagalovsky, B. Horovitz, Y. Avishai, and A. W. W. Ludwig, Thermal metal in network models of a disordered two-dimensional superconductor, Phys. Rev. B 65, 012506 (2001).

[67] C. R. Laumann, A. W. W. Ludwig, D. A. Huse, and S. Trebst, Disorder-induced Majorana metal in interacting non-Abelian anyon systems, Phys. Rev. B 85, 161301(R) (2012).

[68] V. Lahtinen, A. W. W. Ludwig, J. K. Pachos, and S. Trebst, Topological liquid nucleation induced by vortex-vortex interactions in Kitaev's honeycomb model, Phys. Rev. B 86, 075115 (2012).

[69] C. N. Self, J. Knolle, S. Iblisdir, and J. K. Pachos, Thermally induced metallic phase in a gapped quantum spin liquid: Monte carlo study of the Kitaev model with parity projection, Phys. Rev. B 99, 045142 (2019).

[70] A. Weisse, G. Wellein, A. Alvermann, and H. Fehske, The kernel polynomial method, Rev. Mod. Phys. 78, 275 (2006).

[71] C. Wang, A. Vishwanath, and B. I. Halperin, Topological order from disorder and the quantized hall thermal metal: Possible applications to the $v=5 / 2$ state, Phys. Rev. B 98, 045112 (2018).

[72] D. J. Thouless, M. Kohmoto, M. P. Nightingale, and M. den Nijs, Quantized Hall Conductance in a Two-Dimensional Periodic Potential, Phys. Rev. Lett. 49, 405 (1982).

[73] Q. Niu, D. J. Thouless, and Y.-S. Wu, Quantized Hall conductance as a topological invariant, Phys. Rev. B 31, 3372 (1985).

[74] T. Fukui, Y. Hatsugai, and H. Suzuki, Chern numbers in discretized Brillouin zone: Efficient method of computing (spin) Hall conductances, J. Phys. Soc. Jpn. 74, 1674 (2005).

[75] D. P. Arovas, R. N. Bhatt, F. D. M. Haldane, P. B. Littlewood, and R. Rammal, Localization, Wave-Function Topology, and the Integer Quantized Hall Effect, Phys. Rev. Lett. 60, 619 (1988).

[76] F. Evers and A. D. Mirlin, Anderson transitions, Rev. Mod. Phys. 80, 1355 (2008).

[77] B. I. Halperin, Quantized Hall conductance, current-carrying edge states, and the existence of extended states in a twodimensional disordered potential, Phys. Rev. B 25, 2185 (1982).

[78] Y. Huo and R. N. Bhatt, Current Carrying States in the Lowest Landau Level, Phys. Rev. Lett. 68, 1375 (1992).

[79] A. C. Potter and R. Vasseur, Symmetry constraints on manybody localization, Phys. Rev. B 94, 224206 (2016).

[80] G. De Tomasi, F. Pollmann, and M. Heyl, Solving efficiently the dynamics of many-body localized systems at strong disorder, Phys. Rev. B 99, 241114 (2019).

[81] L. M. Robledo, The sign of the overlap of Hartree-FockBogoliubov wave functions, Phys. Rev. C 79, 021302(R) (2009).

[82] M. Fagotti and P. Calabrese, Entanglement entropy of two disjoint blocks in $X Y$ chains, J. Stat. Mech. (2010) P04016. 
[83] M. Wimmer, Efficient numerical computation of the Pfaffian for dense and banded skew-symmetric matrices, ACM Trans. Math. Softw. 38, 1 (2012).

[84] I. Klich, in Quantum Noise, edited by Y. V. Nazarov and Y. M. Blanter (Kluwer, Dordrecht, 2003).

[85] I. Klich, A note on the full counting statistics of paired fermions, J. Stat. Mech. (2014) P11006.

[86] I. L. Aleiner, B. L. Altshuler, and G. V. Shlyapnikov, Finite temperature phase transition for disordered weakly interacting bosons in one dimension, Nat. Phys. 6, 900 (2010).

[87] C. Nayak, S. H. Simon, A. Stern, M. Freedman, and S. Das Sarma, Non-Abelian anyons and topological quantum computation, Rev. Mod. Phys. 80, 1083 (2008).

[88] A. Y. Kitaev, Fault-tolerant quantum computation by anyons, Ann. Phys. (NY) 303, 2 (2003).

[89] C. C. Chamon, C. Mudry, and X.-G. Wen, Gaussian Field Theories, Random Cantor Sets and Multifractality, Phys. Rev. Lett. 77, 4194 (1996).

[90] Y. Hatsugai, X.-G. Wen, and M. Kohmoto, Disordered critical wave functions in random bond models in two dimensions: Random-lattice fermions at $E=0$ without doubling, Phys. Rev. B 56, 1061 (1997).

[91] A. Altland and B. D. Simons, Field theory of the random flux model, J. Phys. A: Math. Gen. 32, L353 (1999).

[92] O. Hart, Y. Wan, and C. Castelnovo, Coherent propagation of quasiparticles in topological spin liquids at finite temperature, Phys. Rev. B 101, 064428 (2020).

[93] A. Russomanno, S. Notarnicola, F. M. Surace, R. Fazio, M. Dalmonte, and M. Heyl, Homogeneous Floquet time crystal protected by gauge invariance, Phys. Rev. Res. 2, 012003(R) (2020).

[94] H. C. Po, L. Fidkowski, A. Vishwanath, and A. C. Potter, Radical chiral Floquet phases in a periodically driven Kitaev model and beyond, Phys. Rev. B 96, 245116 (2017).
[95] I. C. Fulga, M. Maksymenko, M. T. Rieder, N. H. Lindner, and E. Berg, Topology and localization of a periodically driven Kitaev model, Phys. Rev. B 99, 235408 (2019).

[96] L.-M. Duan, E. Demler, and M. D. Lukin, Controlling Spin Exchange Interactions of Ultracold Atoms in Optical Lattices, Phys. Rev. Lett. 91, 090402 (2003).

[97] A. Micheli, G. Brennen, and P. Zoller, A toolbox for latticespin models with polar molecules, Nat. Phys. 2, 341 (2006).

[98] J. Q. You, X.-F. Shi, X. Hu, and F. Nori, Quantum emulation of a spin system with topologically protected ground states using superconducting quantum circuits, Phys. Rev. B 81, 014505 (2010).

[99] M. Sameti and M. J. Hartmann, Floquet engineering in superconducting circuits: From arbitrary spin-spin interactions to the Kitaev honeycomb model, Phys. Rev. A 99, 012333 (2019).

[100] G. Kells, V. Lahtinen, and J. Vala, Kitaev spin models from topological nanowire networks, Phys. Rev. B 89, 075122 (2014)

[101] E. Sagi, H. Ebisu, Y. Tanaka, A. Stern, and Y. Oreg, Spin liquids from Majorana zero modes in a Cooper-pair box, Phys. Rev. B 99, 075107 (2019).

[102] H. Zhang, S. Kim, Y.-J. Kim, H.-Y. Kee, and L. Yang, Ultrafast dynamics of fractional particles in $\alpha-\mathrm{RuCl}_{3}$, arXiv:1908.04807.

[103] J. Knolle and R. Moessner, A field guide to spin liquids, Annu. Rev. Condens. Matter Phys. 10, 451 (2019).

[104] Y. Motome and J. Nasu, Hunting Majorana fermions in Kitaev magnets, J. Phys. Soc. Jpn. 89, 012002 (2020).

[105] M. Ye, R. M. Fernandes, and N. B. Perkins, Phonon dynamics in the Kitaev spin liquid, Phys. Rev. Research 2, 033180 (2020).

[106] A. Metavitsiadis and W. Brenig, Phonon renormalization in the Kitaev quantum spin liquid, Phys. Rev. B 101, 035103 (2020). 